\title{
Advanced Modeling of Prompt Fission Neutrons and Gamma Rays
}

\author{
P. Talou ${ }^{\mathrm{a}}$ and T. Kawano \\ Nuclear Physics Group, Theoretical Division, Los Alamos National Laboratory, Los Alamos, NM 87545, USA
}

\begin{abstract}
Prompt fission neutrons and gamma rays are computed using a Monte Carlo treatment of the statistical evaporation of the excited primary fission fragments. The assumption of two fragments in thermal equilibrium at the time of neutron emission is addressed by studying the neutron multiplicity as a function of fragment mass. Results for the neutron-induced fission of ${ }^{235} \mathrm{U}$ are discussed, for incident neutron energies from 0.5 to $5.5 \mathrm{MeV}$. Recent experimental data on the fission fragment yields as a function of mass and total kinetic energy are used as input data.
\end{abstract}

\section{Introduction}

Prompt fission neutrons and $\gamma$-rays are indirect probes of nuclear configurations near the scission point. Studying them in detail can reveal some interesting characteristics of the fission process itself. In particular, it shines some light on how the total excitation energy available in the system is shared between the light and heavy fragments. The initial spin distribution of the primary fission fragments, about which very little is known, can also be studied through an analysis of the competition between prompt neutron and $\gamma$-ray emission.

Until recently, only average quantities, average spectrum and average multiplicity, were considered in the evaluation of prompt fission neutrons for application needs. At this level of sophistication, important physics questions cannot be addressed. Going beyond averages, one can learn much more about the fission process, in particular near the scission point, and provide applications with new detailed information, such as energy and angular correlations between emitted neutrons.

The present paper discusses Monte Carlo simulations of the statistical evaporation of primary fission fragments using Hauser-Feshbach type equations. Results for the neutroninduced fission of ${ }^{235} \mathrm{U}$ are shown. In particular, the question of the sharing of the total excitation energy between the light and heavy fragments is addressed.

\section{Prompt Fission Neutrons and Gamma Rays}

In the present work, prompt fission neutrons are defined as neutrons emitted within $\sim 10^{-13}$ seconds of the time of scission. Among them, one can distinguish between prescission, scission and post-scission neutrons. Pre-scission neutrons are those emitted during the descent from saddle

\footnotetext{
a e-mail: talou@lanl.gov
}

to scission points. Scission neutrons would be those emitted right at the point of breakup of the parent nucleus into two fission fragments. Finally, post-scission neutrons are emitted once the fission fragments are well formed. Because of the extreme difficulty in separating those three neutron sources experimentally, we will not distinguish them for the present study. In fact, we will assume that all neutrons are emitted from fully accelerated fragments.

Prompt fission $\gamma$-rays are also emitted by the excited primary fission fragments in competition with neutron emission. However, they are usually emitted by fragments whose residual excitation energy lies below the neutron separation energy, hence when the only open decay channel is through $\gamma$-ray emission. In the present calculations, it is assumed that $\gamma$-rays are emitted posterior to the neutrons.

\section{Open Questions}

There remain quite a lot of open questions related to prompt fission neutrons and $\gamma$-rays. For obvious reasons, a few fissioning systems have been studied thoroughly, while many other actinides, not as important for applications, have been neglected. But even in the case of one of the most well known system, uranium-235, the exact values of the prompt fission neutron spectrum for thermal incident neutrons remain controversial [1]. A recent measurement by Hambsch [2] confirmed some previous differential measurements, and as such confirmed the discrepancy observed with integral data.

Important questions have been raised recently on the validity of the Los Alamos model (see below) for lowoutgoing neutron energies, below $\sim 500 \mathrm{keV}$. Some experimental measurements suggest that the true spectrum lies above the Los Alamos predictions, and the discrepancy cannot shrunk even with more advanced versions of the model. Some authors have suggested that scission neutrons could be responsible for the deviation. However, the question remains open, as measurements of low-energy neutrons are notoriously difficult to perform. 


\section{EPJ Web of Conferences}

While a fair number of PFNS measurements have been performed at thermal incident neutron energy, very few data exist as a function of incident neutron energy. Such data would be particularly useful to study the fission process, and not only cross sections, as a function of the excitation energy of the compound system.

Experimental data on prompt $\gamma$-rays are even rarer. New experimental measurements of prompt fission gamma-ray spectrum, multiplicity, and more exclusive observable should be encouraged, as a comprehensive understanding of the decay of the fission fragments cannot be achieved without them. Studying the competition of collective $\gamma$-rays with prompt neutrons is of particular importance to study the initial spin distribution in the fragments. Such quantity could then be used to strongly constrain theoretical model calculations that aim at predicting the configurations of the nascent fragments.

\section{Theoretical Modeling}

The theoretical modeling of prompt fission neutrons started early on with the work of J.Terrell [3]. As of today, the most reliable nuclear data files present in existing evaluated libraries contain prompt fission neutron spectrum data evaluated using the so-called Los Alamos (LA) or MadlandNix model [4] at its core. In many instances, a simpler representation based on a Watt spectrum is even used. Various developments starting from the initial equations of the LA model have been derived over the years (see for instance Refs. $[5,6]$ ), but they all rely on the same basic equations and assumptions.

\subsection{Los Alamos Model}

Here we only discuss the main assumptions of the Los Alamos model [4]. In this model, only a small subset of the fission fragments produced in the reaction are considered, at and around the top of the fragment yields. The fragments are formed with a range of excitation energies, represented by a triangular distribution of nuclear temperatures. A temperature is obtained from an excitation energy through the use of the Fermi-gas relation $E^{*}=a T^{2}$, where $a$ is the level density parameter of the fragment. Prompt neutrons are assumed to be emitted from a Weisskopf evaporation spectrum at temperature $T$. Taking into account the boost due to kinematic energy from the fragments, the average prompt neutron spectrum in the laboratory is finally obtained.

Three important assumptions are made in the LA model: i) neutrons are emitted from fully accelerated fission fragments only; ii) the temperatures of the light and heavy fragments are both equal to the temperature in the parent fissioning system; iii) the same number of neutrons is emitted from the light as from the heavy fragment.

The LA model has been used very successfully over the years to compute the average prompt neutron spectrum for many fissioning systems and for incident neutron energies up to $\sim 20 \mathrm{MeV}$, with a very limited set of adjustable parameters.

\subsection{Monte Carlo Approach}

Although very successful, the LA model cannot go beyond what it is was originally intended for: calculating average quantities. To dig deeper into our understanding of the nuclear fission process, more detailed data need to be predicted and compared to experimental data. In addition, practical applications of exclusive observable are beginning to emerge (e.g., non-proliferation). That is exactly the purpose of the Monte Carlo approach discussed here, and already implemented in earlier calculations [7].

Assuming an initial distribution of primary fission fragments as a function of mass, charge and excitation energy $Y(A, Z, U)$, one can perform Monte Carlo simulations of the evaporation stage of those fragments by emission of neutrons and $\gamma$-rays leading to a distribution of fission products. The now familiar Hauser-Feshbach equations can be used to compute the probabilities of neutrons and $\gamma$-rays to be emitted at each stage of the evaporation cascade. The result is a record file containing a large number of Monte Carlo histories, the analysis of which resembles the analysis of experimental data sets. In this type of calculations, an exclusive observable can be inferred rather simply. As an example, the exclusive spectrum of prompt neutrons for which 2 and only 2 neutrons are emitted can be obtained simply by collecting the kinetic energies of the neutrons emitted only in the case the total neutron multiplicity for an event is equal to 2 . A similar result could be obtained with well-known deterministic approaches, but with rather cumbersome book-keeping techniques [8].

Other exclusive quantities of interest are: neutron multiplicity distribution $P(v)$; average neutron multiplicity as a function of fragment mass $\bar{v}(A)$; neutron-neutron angular and energy correlations; $\gamma-\gamma$ correlations; etc.

It is important to note that this approach does not address the question of scission neutrons any more than the LA model does, as all neutrons are assumed to be emitted from the fully accelerated fragments. However, the Monte Carlo approach provides exclusive data that can be compared to experimental results, leading to a better understanding of the physics near the scission point. In this sense only does it provide an indirect answer to the question of scission neutrons.

In the following, the question of thermal equilibrium between the light and heavy fragments is addressed. For a given pair of fission fragments and for a given value of the average total kinetic energy $\langle T K E\rangle$, the average total excitation energy $\langle T X E\rangle$ available is easily inferred using

$$
\langle T X E\rangle=Q_{f}-\langle T K E\rangle,
$$

where $Q_{f}$ is the $Q$-value of the fission reaction, and is simply given by a difference of nuclear masses. What is not known however is how $\langle T X E\rangle$ is shared among the light and heavy fragments. In the original LA model, both fragments are assumed to be in thermal equilibrium at scission. Although a reasonable assumption, it does not preclude the possibility of a collective deformation energy being different for the light and heavy nascent fragments. At the time of neutron emission, once the fragments are fully accelerated, these collective energies for both fragments would 
have dissipated into intrinsic energies. At that time, there is little reason for both nuclear temperatures to remain identical.

To study this effect, let us introduce the parameter

$$
R_{T}=\frac{\left\langle T_{l}\right\rangle}{\left\langle T_{h}\right\rangle},
$$

where $\left\langle T_{l}\right\rangle$ and $\left\langle T_{h}\right\rangle$ are the temperatures in the light and heavy fragments, respectively. The numerical results shown below will be analyzed as a function of this parameter $R_{T}$. To simplify the calculations, no neutron-gamma competition is considered in this work, and $\gamma$-rays are assumed to be emitted only when the residual energy is too low for further neutron emission. Additional work is in progress to lift this assumption [8].

\section{Numerical Results}

We have performed Monte Carlo simulations for the neutron induced fission of ${ }^{235} \mathrm{U}$ for incident neutron energies ranging from 0.5 to $5.5 \mathrm{MeV}$. Experimental data from Hambsch [9] are used for the primary fission fragment yields $Y(A, T K E)$. Figure 1 shows the experimental yields at 0.5 $\mathrm{MeV}$ incident neutron energy.

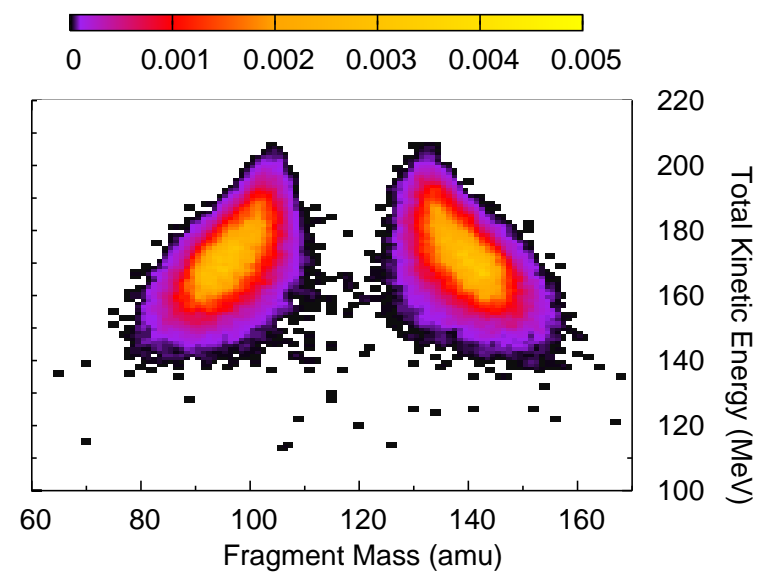

Fig. 1. Experimental fission fragment yields for the $\mathrm{n}(0.5$ $\mathrm{MeV})+{ }^{235} \mathrm{U}$ reaction (Source: F.J.-Hambsch [9]).

We sampled these experimental fission fragment yields, and using $R_{T}$ as a free parameter, computed the initial conditions for the evaporation of fragments $(A, Z, U)$. Following all neutron emissions until the residual energy is lower than the neutron binding energy, we accumulated a large number of Monte Carlo histories that were then analyzed.

The average prompt fission neutron spectrum (PFNS) calculated in this way is shown in Fig. 2 for three different values of $R_{T}: 1.0,1.2$, and 1.4 . We have considered only $R_{T}$ values greater than 1.0 as experimental evidence suggest that more neutrons are emitted from the light than from the heavy fragments (see discussion below). Compared to the evaluated results using the LA model, such as ENDF/BVII.0 and JENDL-Actinoid results, and to the experimental data points by Johansson [10], the Monte Carlo calculated spectra are too soft, dropping below the data points for outgoing neutron energies above about 2-3 MeV. This result holds true for all values of the $R_{T}$ parameter, although the result for $R_{T}=1.4$ is in relatively good agreement with the evaluated files.

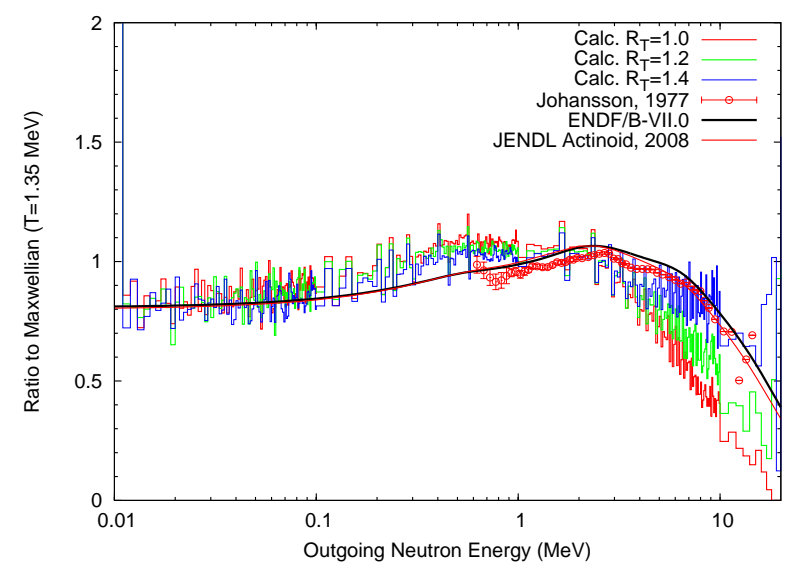

Fig. 2. Calculated prompt fission neutron spectrum for the $\mathrm{n}+{ }^{235} \mathrm{U}$ reaction, at $E_{i n c}=0.5 \mathrm{MeV}$. Calculations were done for three different values of the $R_{T}$ parameter: 1.0,1.2, and 1.4.

Monte Carlo calculations allow the exploration of physical observables beyond average quantities. Figure 3 shows the average neutron multiplicity as a function of fragment mass $\bar{v}(A)$. The well-known saw-tooth behavior is well reproduced by the calculations, and is primarily due to nuclear structure effects, embodied in the $a$ level density parameter. In the present calculations, the Gilbert-CameronIgnatyuk formalism was used to describe level densities in the continuum for each fission fragment. Even more interesting is the evolution of the results as a function of $R_{T}$. For $R_{T}=1.0$ (red circles), too few neutrons are emitted from the light fragments, while too many are emitted from the heavy fragments, calling for a $R_{T}$ value higher than 1.0. In fact, the results obtained with $R_{T}=1.2$ are in very good agreement with experimental data.

Another quantity of interest in the neutron multiplicity distribution $P(v)$, shown in Fig. 4. The agreement with the experimental data from Boldeman [11] is only fair.

The same study can be performed for higher incident neutron energies, but below the onset of second-chance fission, for which experimental yields have been measured [9]. At $5.5 \mathrm{MeV}$, the mass symmetric region starts to fill up, and $\langle T K E\rangle$ is lowered. Hence, the average total excitation energy $\langle T X E\rangle$ available in the system increases. Therefore, we can expect more neutrons to be emitted at this incident energy not only because of the additional kinetic energy of $5.0 \mathrm{MeV}$ but also because of the reduction of $\langle T K E\rangle$.

The average neutron multiplicity as a function of fragment mass $\bar{v}(A)$ is shown in Fig. 5 along with experimental data from Müller [12]. In this case, the results for $R_{T}=1.0$ agree best with the experimental data. It can be understood 
as follows: as the incident neutron energy increases, the role of shell effects diminishes, and the ratio of collective energies stored in the light and heavy fragments tends toward one. Also, the additional kinetic energy present in the system is directly present as an intrinsic excitation energy in the fissioning system. In this case, the assumption of phase-space sharing of the energy among the two fragments is most convincing. Both effects would tend toward $R_{T} \simeq 1.0$.

Other fissioning systems have been studied, but will not be reported here for lack of space. A longer publication is in preparation.

\section{Conclusion}

Prompt fission neutrons represent very powerful probes of the nuclear fission process near the scission point. Along with prompt $\gamma$-rays (not addressed here) they can be used to study the configurations of the fission fragments very close to their birth place, and better understand how the total excitation energy available in the system gets transferred to intrinsic excitation in the fragments. Average observables such as the average PFNS $N(E)$ or neutron multiplicity $\bar{v}$ are not sufficient to provide clear answers to those fundamental questions, but more exclusive quantities such as $P(v)$ and $\bar{v}(A)$ may. The Monte Carlo studies presented here are a very promising tool to study the physics near scission and provide new results useful for applications as well.

\section{Acknowledgments}

The authors would like to thank Dr. F.-J. Hambsch for sending his important data on fission fragment yields.

\section{References}

1. D.G. Madland et al., Fission Neutron Spectra of Uranium-235, OECD-WPEC Subgroup 9 Report (2003).

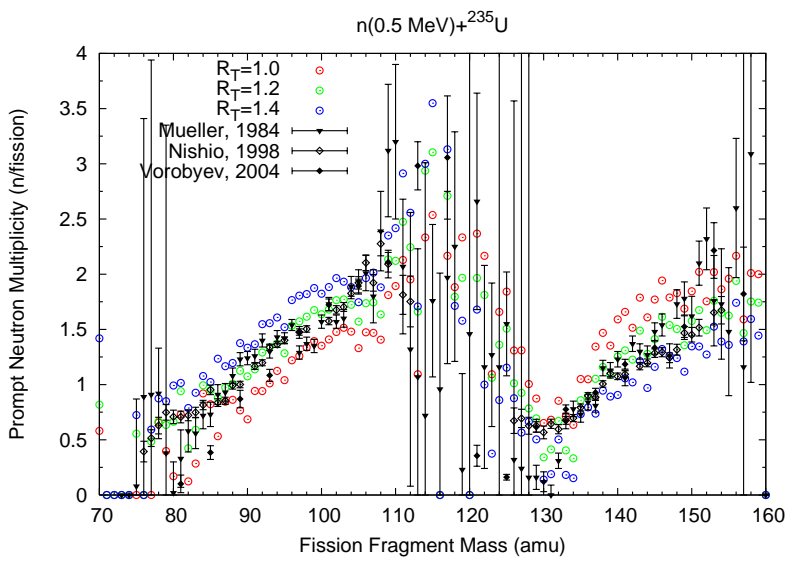

Fig. 3. Average neutron multiplicity as a function of fission fragment mass.

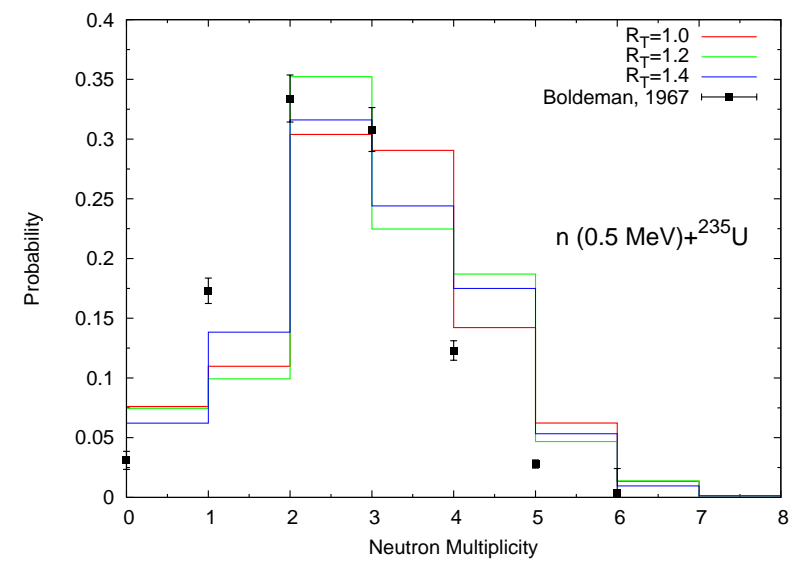

Fig. 4. Prompt fission neutron multiplicity distribution calculated for $0.5 \mathrm{MeV}$ incident neutrons on ${ }^{235} \mathrm{U}$.

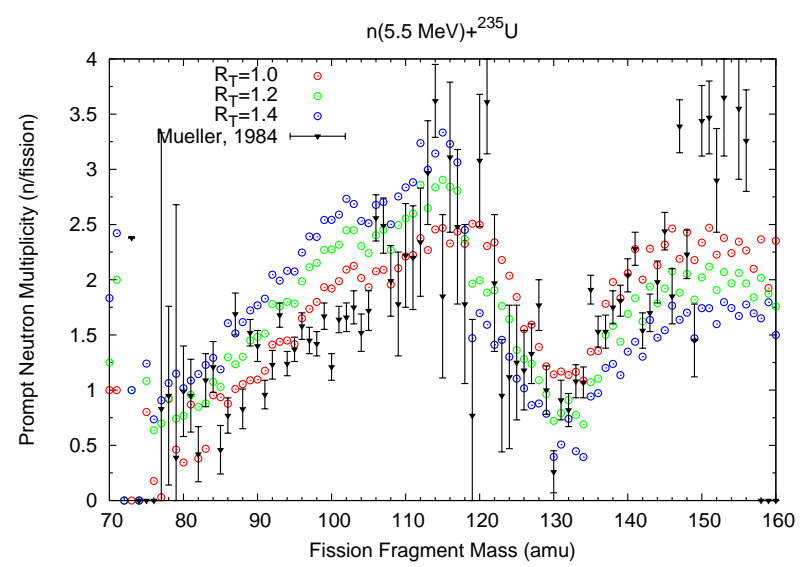

Fig. 5. Same as Fig. 3 but for $5.5 \mathrm{MeV}$ incident neutron energy.

2. F.-J. Hambsch, this Conference.

3. J. Terrell, Phys. Rev. 113 (1959) 527-541.

4. D.G. Madland and J.R. Nix. Nucl. Sci. Eng. 81 (1982) 213-271.

5. T. Ohsawa, INDC (NDS) report 251 (1991).

6. G. Vladuca and A. Tudora, Ann. Nucl. Energy 28 (2001) 419.

7. S. Lemaire, P. Talou, T. Kawano, M.B. Chadwick and D.G. Madland, Phys. Rev. C 72 (2005) 024601.

8. T. Kawano, P. Talou, M.B. Chadwick and T. Watanabe, submitted to J.Nucl.Sci.Tech. (2009).

9. F.-J. Hambsch (2007) private communication.

10. P.I. Johansson and B. Holmqvist, Nucl. Sci. Eng. 62 (1977) 695.

11. J.W. Boldeman and A.W. Dalton, AAEC/E172 (1967).

12. R. Müller, A.A. Naqvi, F. Käppeler, and F. Dickmann, Phys. Rev. C 29 (1984) 885.

13. K. Nishio, Y. Nakagome, H. Yamamoto, I. Kimura, Nucl. Phys. A632 (1998) 540. 